\title{
Evaluation of the Synergistic Effect of EDTA-Functionalized Chitosan Nanoparticles on Imipenem Delivery in Pseudomonas aeruginosa Carbapenem-Resistant Strain AG1
}

\author{
Marilyn Porras-Gómez ${ }^{1}$, José Vega-Baudrit ${ }^{1}$, Fernando García ${ }^{2}$, \\ Santiago Núñez-Corrales ${ }^{1}$, Sergio Madrigal-Carballo ${ }^{3}$
}

${ }^{1}$ National Nanotechnology Laboratory (LANOTEC), National Center for Advanced Technology Studies (CeNAT), San José, Costa Rica

${ }^{2}$ Centro de Investigaciones en Enfermedades Tropicales (CIET), Facultad de Microbiología, Universidad de Costa Rica, San Pedro, Costa Rica

${ }^{3}$ National Center for Biotechnological Innovations (CENIBiot), National Center for Advanced Technology Studies (CeNAT), San José, Costa Rica

Email: marilyn.ucr@gmail.com

How to cite this paper: Porras-Gómez, M., Vega-Baudrit, J., García, F., Núñez-Corrales, S. and Madrigal-Carballo, S. (2018) Evaluation of the Synergistic Effect of EDTA-Functionalized Chitosan Nanoparticles on Imipenem Delivery in Pseudomonas aeruginosa Carbapenem-Resistant Strain AG1. Journal of Biomaterials and Nanobiotechnology, 9, 64-78. https://doi.org/10.4236/jbnb.2018.91006

Received: October 13, 2017

Accepted: January 8, 2018

Published: January 11, 2018

Copyright $\odot 2018$ by authors and Scientific Research Publishing Inc. This work is licensed under the Creative Commons Attribution International License (CC BY 4.0).

http://creativecommons.org/licenses/by/4.0/ (c) (i) Open Access

\begin{abstract}
Metallo- $\beta$-lactamases are bacterial zinc-dependent enzymes involved in the hydrolysis of $\beta$-lactamic antibiotics representing the main cause of bacterial resistance to carbapenems, drugs of last resort for treating infections caused by multiresistant bacteria. We elaborated the hypothesis that it is possible to inhibit the enzymatic activity of metallo- $\beta$-lactamases by lowering the availability of zinc in the extracellular medium using metal chelating agents such as EDTA carried on nanoparticles. Chitosan, as linear cationic polysaccharide is frequently used in biomedical and pharmaceutical applications, has been studied as a biocompatible encapsulating agent in drug delivery systems and is an ideal transport agent for bioactive molecular complexes in antibiotic applications due to its ability to associate with negatively charged substances. We developed novel nanoparticles using chitosan as a transport matrix for $\beta$-lactamic antibiotics. Nanoparticles were synthesized according to the ion gelation method using tripolyphosphate as crosslinking agent. Nanoparticles were functionalized by the adsorption of EDTA, which acts as complexifying agent for $\mathrm{Zn}^{2+}$ ions causing inhibition of metallo- $\beta$-lactamases activity. We evaluate the antimicrobial effects of EDTA-functionalized nanoparticles with an imipenem cargo on the clinical isolate $P$. aeruginosa AG1, a carbapenem-resistant high-risk clone ST-111 carrying both $b l_{a_{\mathrm{IMP}-18}}$ and bla $a_{\mathrm{VIM}-2}$ metallo- $\beta$-lactamases genes.
\end{abstract}




\section{Keywords}

Chitosan, EDTA, Nanoparticles, Drug-Resistant, Imipenem

\section{Introduction}

\subsection{Clinical Relevance of Pseudomonas aeruginosa}

Pseudomonas aeruginosa, a Gram-negative bacterial pathogen bacterium with extraordinary physiological and metabolic versatility found mostly in water reservoirs, causes severe nosocomial and community acquired infections at a variety of body sites including the urinary tract, surgical or burn wounds and the lower respiratory tract [1]. Patient groups at risk for acquisition of $P$. aeruginosa infections include those with hereditary diseases such as cystic fibrosis and burn patients, ones hospitalized in intensive care units and those undergoing mechanical ventilation, and patients immunosuppressed by certain diseases like cancer and AIDS. Although antibiotic therapy has been considerably improved in the management of infectious diseases in general, many $P$. aeruginosa infections cannot be fully treated or eradicated by the application of drugs and can thus establish chronic infections [2] [3].

$P$. aeruginosa infections are a serious therapeutic challenge due to the difficulty in their treatment and control: acquired mechanisms of resistance found commonly in $P$. aeruginosa isolates often render ineffective known disinfectants and antibiotics [1] [4]. The global emergence of multiresistant $P$. aeruginosa strains has been widely acknowledged as an extraordinary threat to public health [4] [5] [6].

$\beta$-lactamases confer significant antibiotic resistance to their bacterial hosts by hydrolyzing the amide bond of the four-membered $\beta$-lactam ring. These enzymes are especially important in $P$. aeruginosa as they constitute the major defense mechanism against $\beta$-lactam antibiotics such as carbapenems [7].

Class B enzymes, metallo- $\beta$-lactamases (MBLs), require a zinc divalent ion in order to perform their activity. With the aim of blocking the activity of MBLs, the search for activity inhibitors and deactivators along with their coupling with a $\beta$-lactam antibiotic has been proposed. The latter in promising in the sense of finding adequate molecules that can be added to carbapenems, resulting in combinations with the ability to overcome resistance mediated by metallo- $\beta$-lactamases [8]. Given that beta-lactams are the last therapeutic line of defense against $P$. aeruginosa infections, their resistance to carbapenems is of the highest concern.

\subsection{Resistance Profile of $P$, aeruginosa Strain AG1}

A total of 198 non-duplicated $P$. aeruginosa AG1 isolates from a study in Costa Rica were evaluated for their susceptibility to beta-lactams, aminoglycosides and fluoroquinolones. From the sample, $63.1 \%$ were categorized as carbapenem-resistant and $88.8 \%$ of the carbapemen-resistant isolates were also resistant to ceftazidime, cefepime, 
aztreonam, ticarcillin/clavulanic acid, amikacin, gentamicin, tobramycin, ciprofloxacin and gatifloxacin. $81.6 \%$ of the carbapenem-resistant isolates showed MBL activity. The blaIMP and blaVIM genes were present in $94.1 \%$ of the MBL-producing isolates [9].

\subsection{Design of Chitosan Nanoparticles for Synergistic Antimicrobial Activity}

Chitosan is a linear polysaccharide that is composed of randomly distributed $\mathrm{D}$-glycosamine and $\mathrm{N}$-acetyl-glycosamine units linked in a $\beta(1 \rightarrow 4)$ manner [10]. Chitosan is used in multiple biomedical and pharmaceutical applications because of its bioavailability, nontoxicity, biocompatibility, biodegradability and high positive charge density. Due to the advantages it offers, the use of chitosan for obtaining cationic polymeric nanoparticles as delivery systems has been extensively studied [11]. Research showed that nanoparticles were able to protect the drugs from degradation and controlled the release of the encapsulated or adsorbed drugs [12] [13].

These nanoparticles can achieve a size of up to $50 \mathrm{~nm}$ and a positive surface charge (reported as $\zeta$ potential) between $+20 \mathrm{mV}$ and $+60 \mathrm{mV}$ [13]. Chitosantripolyphosphate nanoparticles are intended for in vivo administration, so that no harmful residue exists that may compromise the safety of the organism to which they are delivered. Chitosan nanoparticles (CHT NPs) synthesis through the ionic gelation meets this requirement, since the method does not require the addition of any organic solvents, thus avoiding the problem of elimination of residues prior to delivery into living organisms. The ionic gelation involves a complexation between the negative and positive charges of tripolyphosphate (TPP) and chitosan (CHT), respectively, behaving as a metastable thermodynamic system [14].

Ethylenediaminediacetic acid-disodium (EDTA) is a polyamino carboxylic acid and popularly known as chelating agent. The $\mathrm{NH}^{3+}$ moieties present in the quitosan were reported to react with COO- moieties of EDTA to form ionic EDTA-CHT complex. The EDTA-CHT was reported to show antimicrobial activity against Gram-negative and Gram-positive bacteria [15] [16].

In the present study, EDTA-CHT nanopartices (EDTA-CHT NPs) were synthesized by an ionic gelation cross-linking method. Imipenem, a carbapenem, were loaded into the nanoparticles. The physicochemical properties of EDTA-CHT NPs and antimicrobial capability of imipenem-loaded EDTA-CHT NPs was also investigated against carbapenem-resistant $P$. aeruginosa.

\section{Materials and Methods}

\subsection{Chemicals}

Chitosan with molecular weight between 100,000 - 300,000 (ACROS Organics ${ }^{\mathrm{TM}}$ ), sodium tripolyphosphate (Fischer scientific) and glacial acetic acid (J.T.Baker ${ }^{\circledR}$ ) were used. Ultrapure water was obtained using the Milli-Q A10 system (Milli- 
pore). Imipenem monohydrate $\geq 98 \%$ (Sigma-Aldrich) was used as the antimicrobial agent. Disodium EDTA (J.T.Baker ${ }^{\circledR}$ ) were used.

\subsection{Bacterial Strains}

P. aeruginosa AG1, carrier of the bla $a_{\mathrm{IMP}-18}$ and bla $a_{\mathrm{VIM}-2}$ genes with expression of metallo- $\beta$-lactamase activity, was used as model for this study. $P$. aeruginosa PAO1 were used as control strains due to their susceptibility to carbapenems [9] [17]. Bacterial culture medium used was Lysogenic Broth.

\subsection{Synthesis of Chitosan-Tripolyphosphate Nanoparticles}

Chitosan-tripolyphosphate nanoparticles were produced using a modified ionic gelation method. Briefly, chitosan was dissolved at $5 \mathrm{mg} / \mathrm{ml}$ in $2 \% \mathrm{v} / \mathrm{v}$ acid acetic solution at $\mathrm{pH}=5$. Tripolyphosphate was dissolved in ultrapure water to obtain a $1.2 \mathrm{mg} / \mathrm{ml}$ concentration. $1 \mathrm{ml}$ of tripolyphosphate solution was added dropwise to $1.5 \mathrm{ml}$ of chitosan solution and was magnetically stirred at 500 stock/min for $1 \mathrm{~h} \mathrm{[18]} \mathrm{[19].}$

\subsection{Encapsulation of Imipenem}

$1 \mathrm{ml}$ of imipenem (dissolved in a solution of MOPS, ethylene glycol and water 2:1:1) at different concentrations was added at constant stirring before polymer cross-linking. Later addition of sodium tripolyphosphate was required for encapsulation during ionic gelation [19].

\subsection{Surface Functionalization of Nanoparticles}

A concentration gradient of EDTA was tested from 0.39 to $125 \mathrm{mM}$. We chose a concentration of EDTA for CHT NPs synthesis of $3 \mathrm{mM}$ for surface functionalization. Surface functionalization of CHT NPs with chelating agent EDTA was carried out by adding $1 \mathrm{ml}$ of EDTA at a concentration of $5 \mathrm{mg} / \mathrm{ml}$ after ionic gelation, the solution was magnetically stirred at $500 \mathrm{stock} / \mathrm{min}$ for $30 \mathrm{~min}$ more. The resulting reaction is acid-base leading to the formation of an amide bond between the free amino groups $\left(-\mathrm{NH}_{2}\right)$ in chitosan and the carboxyl groups $(-\mathrm{COOH})$ in EDTA [14] [15].

\subsection{Fourier Transform Infrared Analysis}

The structural features of nanoparticles were estimated by Fourier transform infrared (FTIR) Thermo Scientific, Nicolet 6700. The samples for FTIR analysis are prepared by grinding the dry blended CHT and EDTA-CHT NPs using an attenuated total refraction.

\subsection{Determination of Particle Size and $\zeta$ Potential}

Determination of particle size (apparent hydrodynamic diameter) was performed by dynamic light scattering (DLS), polydispersity index value (size distribution) and surface electric charge, reported as $\zeta$ potential or electrophoretic mobility [13]. 


\subsection{Morphology Analysis of Chitosan Nanoparticles}

In order to study the morphology of filtered $(0.2 \mathrm{um})$ and diluted samples (1/10) of nanoparticles, topographic images of CHT-NPs were taken on a multimode atomic force microscope (AFM) Asylum Research MFP-3D. The AFM probes used for this study were rectangular silicon probes with a nominal spring constant of $40 \mathrm{nN} / \mathrm{nm}$. Similarly, image visualization was carried out in a scanning electron microscope (SEM) Hitachi S-3700 with a $15 \mathrm{~nm}$ gold coating on the diluted samples (1/10) using a aluminum base at an acceleration voltage of $15 \mathrm{kV}$ [13].

\subsection{Determination of Encapsulation Efficiency of Chitosan Nanoparticles}

The encapsulation efficiency (EE) of the nanoparticles was determined according to the method described in the previous studies [18]. Imipenem-loaded nanoparticles were separated from aqueous suspension by centrifugation at 11.000 stock $/ \mathrm{min}$ and $22^{\circ} \mathrm{C}$ for 30 minutes, imipenem content in the supernatants was measured by HPLC-DAD S-200 Perkim Elmer. A blank sample was made from nanoparticles without imipenem but treated similarly as the drug-loaded nanoparticles. All samples were measured in triplicate. EE was calculated with the following relation:

$$
E E=F / T \times 100 \%
$$

where, $F$ is the free amount of imipenem in the supernatant and $T$ is total amount of imipenem.

\subsection{In Vitro Release Studies}

The in vitro release studies were carried out in PBS (pH 7.4) as followed: imipenem loaded EDTA-CHT NPs $(1.5 \mathrm{ml})$ and $1.5 \mathrm{ml}$ PBS were incubated at $37^{\circ} \mathrm{C}$ and shaken at 200 stocks/min. Triplicate samples were analyzed at each time step, between 0 and 24 hours. The concentrations of the released imipenem into PBS were determined by HPLC-DAD S-200 Perkin-Elmer.

\subsection{Bacterial Growth Curves}

The spectrophotometrically adjusted inoculum $(100 \mu \mathrm{l})$ of $10^{4}$ bacterial cells was added to each well in the sterile flatbottomed microtiter plate containing the test CHT-NPs. The design of experiments includes duplicated wells of imipenemloaded EDTA-CHT NPs with different concentrations of imipenem, two wells with imipenem as growth inhibition control, two wells containing bacterial suspension with CHT-NPs (growth control) and two wells containing only media (background control) were included in this plate. Dilutions were halved at each consecutive level in the gradient. Optical densities were measured for 12 hours at $37^{\circ} \mathrm{C}$ using a multi-detection microplate reader Biotek Synergy HT at $600 \mathrm{~nm}$ and automatically recorded for each well every $30 \mathrm{~min}$. Turbidimetric growth curves were obtained depending on the changes in the optical density of bacteri- 
al growth for each CHT NP sample and the drug-free growth control.

\section{Results and Discussion}

\subsection{Physicochemical Characterization of Nanoparticles}

The morphological properties of CHT NPs and EDTA-CHT NPs (mean size, $\zeta$ potential and PDI) are indicated in Table 1, where size is measured with respect to diameter. Averages and their standard deviation are reported for each property. According to Table 1, NPs with EDTA increase in size. CHT NPs without EDTA are those with smaller sizes and higher surface charge $50 \mathrm{mV}$, therefore they are stable suspensions [20]. The EDTA surface functionalization CHT NPs increase in size and decrease their surface charge, due to the decrease of free positive charge of chitosan, being attached to EDTA. Encapsulation efficiency was calculated from an initial concentration of imipenem of $1 \mathrm{mg} / \mathrm{ml}$, resulting in a final encapsulated volume of $415.6 \pm 1.1 \mathrm{ug} / \mathrm{ml}$ for a final value of $E E=$ $41.65 \%$.

In present study the results obtained by DLS revealed that the EDTA-CHT NPs are larger than the CHT-NPs ones, possibly due to the EDTA surface adsorption during reaction time. $\zeta$ potential of CHT NPs can greatly influence their stability in suspension by means of electrostatic repulsion between the particles [21]. Our results show $\zeta$ potentials of CHT NPs and EDTA-CHT NPs of 56.07 and $46.63 \mathrm{mV}$ respectively. The latter indicates EDTA-CHT NPs lead to a minor reduction of the particle's $\zeta$ potential.

A batch of samples of CHT-NPs was synthesized for morphological analysis using the AFM. Figure 1 shows different topographic frontal views to better assess surface definition and morphology. The images (Figure 1 and Figure 2) indicate an average size of about $100 \mathrm{~nm}$. The distribution of nanoparticle diameters is reported in Figure 2 after post-processing of the AFM image.

EDTA functionalization has an aggregation effect on CHT NPs, as expected from literature [22]. Additional AFM imaging shows that EDTA-functionalized nanoparticles aggregate in large groups (Figure 3(a)) instead of in a scattered fashion (Figure 3(b)).

The ability of the ionic gelation process to form EDTA-CHT NPs was assessed by employing Fourier transform infrared (FTIR) spectroscopy in order to determine EDTA-CHT interactions. The FTIR spectra of CHT NPs and EDTACHT NPs are shown in Figure 4. FTIR spectroscopy confirmed that in nanoparticles the peaks for N-H bending vibration of amine I at $1600 \mathrm{~cm}^{-1}$ and the amide II carbonyl stretch at $1650 \mathrm{~cm}^{-1}$ shifted to $1540 \mathrm{~cm}^{-1}$ and $1630 \mathrm{~cm}^{-1}$, respectively. Cross-linked chitosan also shows a $\mathrm{P}=\mathrm{O}$ peak at $1170 \mathrm{~cm}^{-1}$. The peak for asymmetric stretch of C-O-C is found at around $1150 \mathrm{~cm}^{-1}$ and the peak at $1317 \mathrm{~cm}^{-1}$ belongs to the $\mathrm{C}-\mathrm{N}$ stretching vibration of type I amine [23].

In the present study, SEM images were taken in order to study the morphological properties and surface appearance of nanoparticles (Figure 5). CHT NPs (Figure 5, column (a)) show a regular surface, spherical shape and approximate 
Table 1. CHT NP size in relation to EDTA concentration during synthesis.

\begin{tabular}{cccccccc}
\hline & \multicolumn{2}{c}{ Size $(\mathrm{nm})$} & \multicolumn{2}{c}{$\zeta(\mathrm{mV})$} & \multicolumn{3}{c}{ PDI } \\
EDTA (\%w/v) & avg & sd & avg & sd & avg & sd \\
0.00 & 127.77 & 4.80 & 56.07 & 0.42 & 0.55 & 0.02 \\
0.50 & 637.10 & 39.14 & 46.63 & 2.54 & 0.76 & 0.03 \\
\hline
\end{tabular}

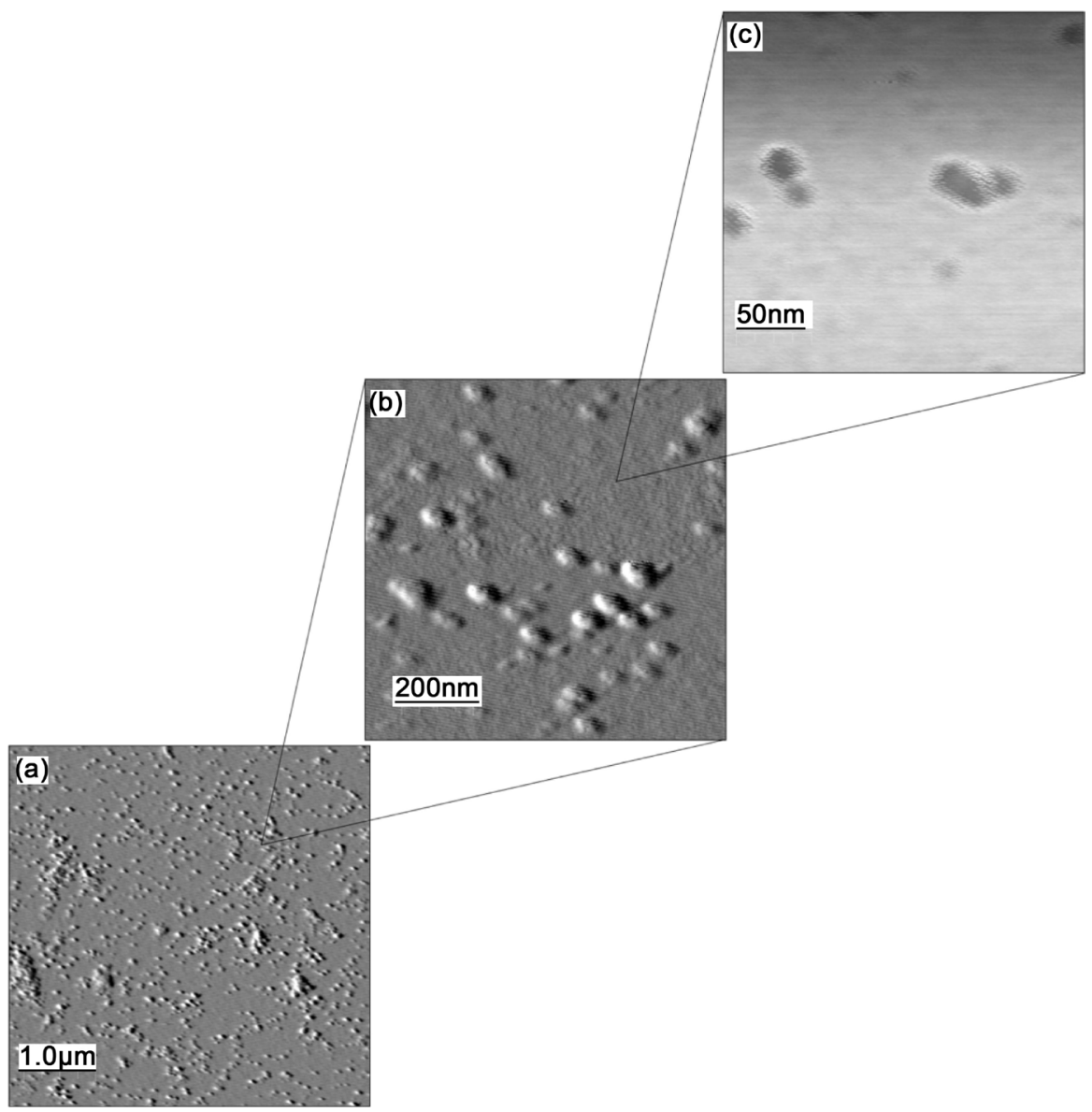

Figure 1. AFM images of CHT NPs. Scan areas are (a) $5 \mu \mathrm{m} \times 5 \mu \mathrm{m}$, (b) $1 \mu \mathrm{m} \times 1 \mu \mathrm{m}$ and (c) $200 \mathrm{~nm} \times 200 \mathrm{~nm}$.

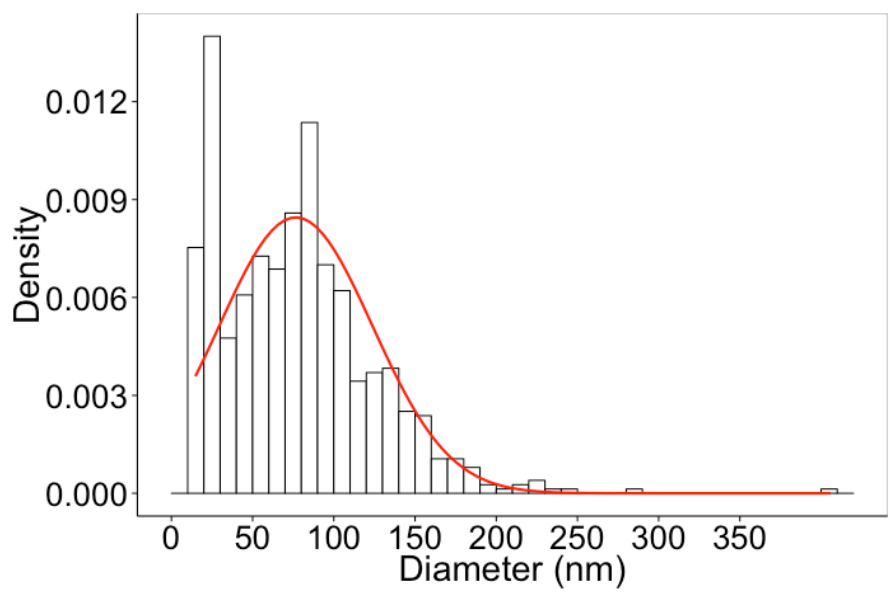

Figure 2. Histogram of CHT NP sizes. Average: $76.6 \pm 47.2 \mathrm{~nm}$. 

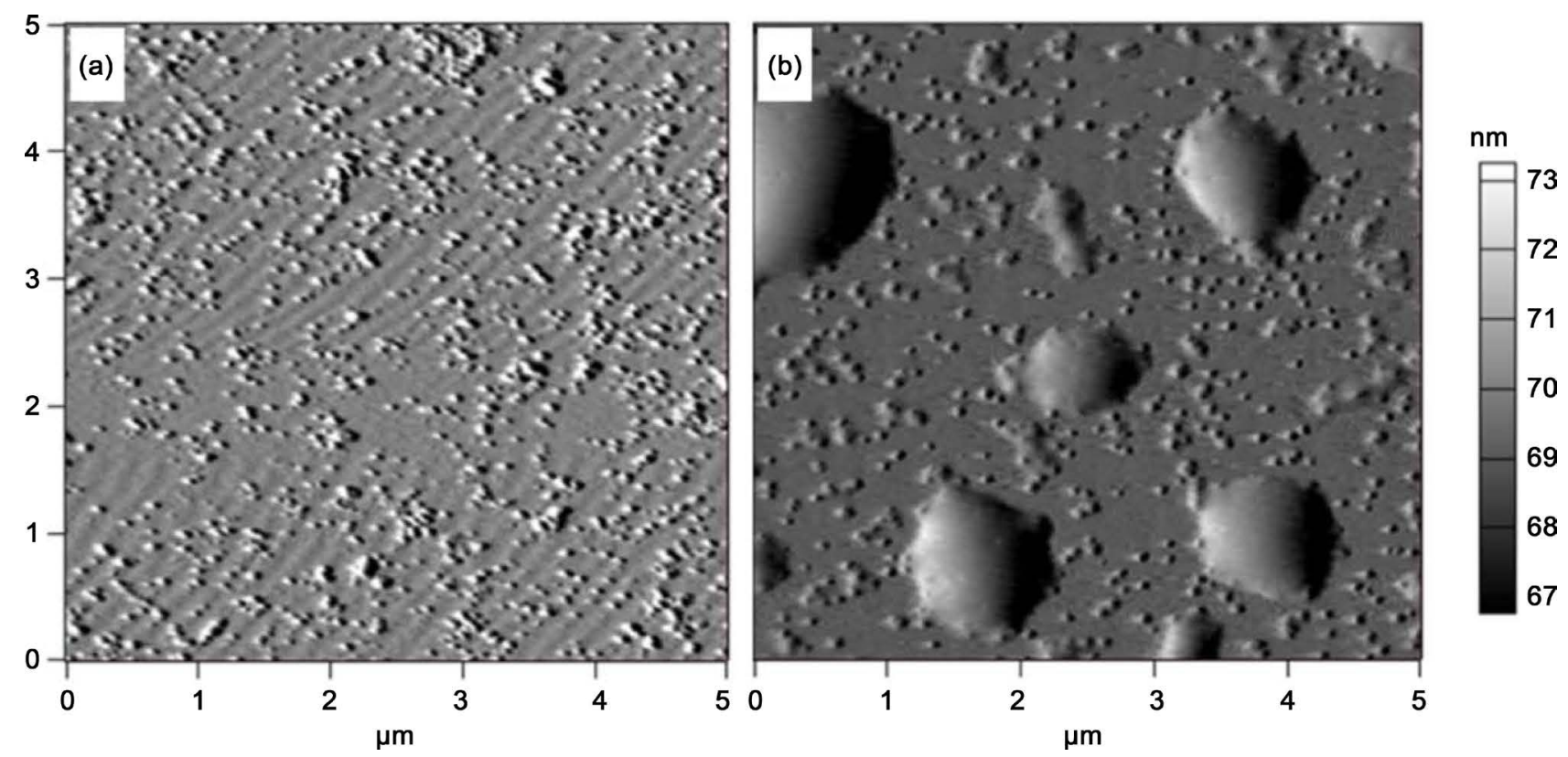

Figure 3. AFM topographic three-dimensional images of nanoparticles. Horizontal, vertical and surface distances are shown. (a) CHT NPs; (b) EDTA-CHT NPs.

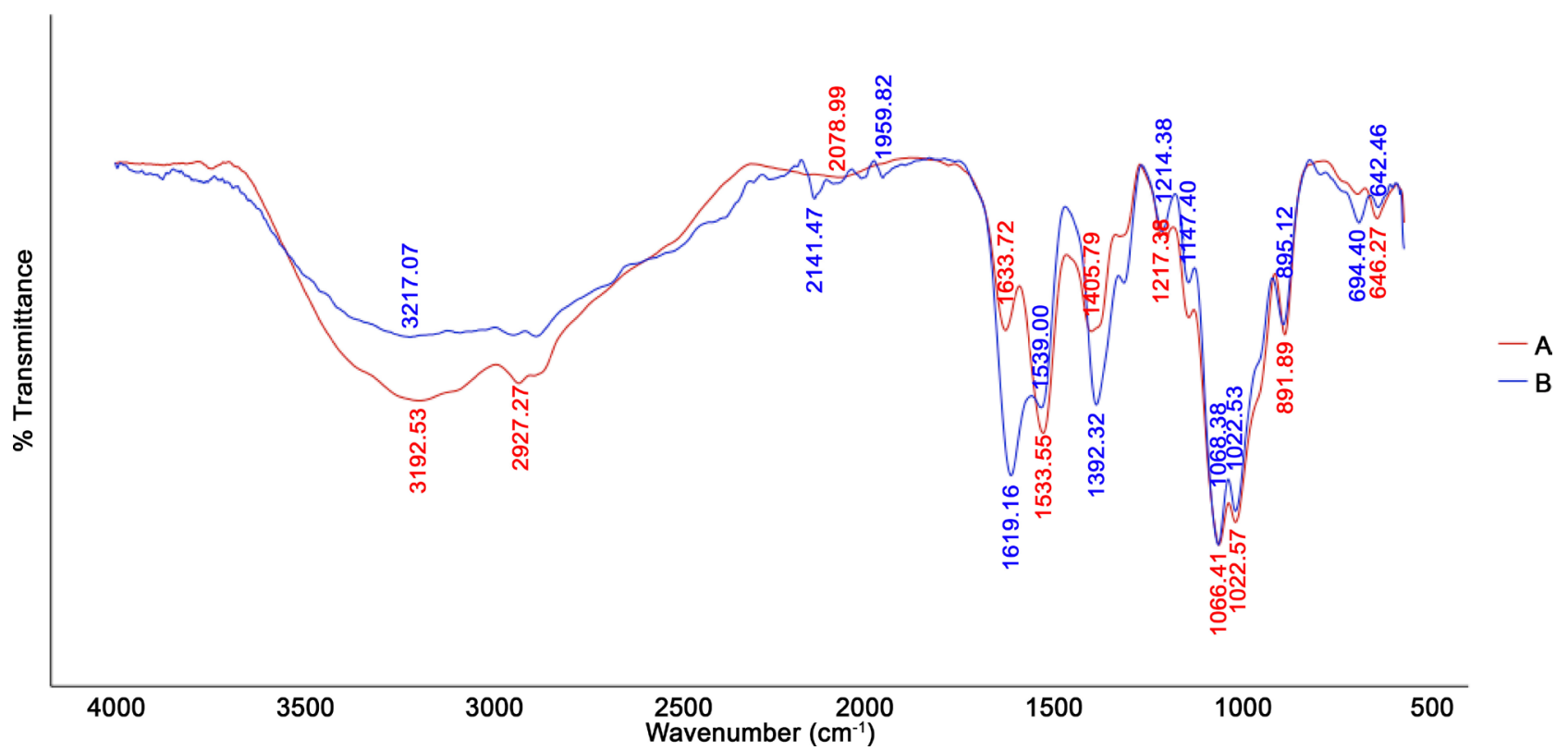

Figure 4. FTIR spectroscopy analysis of (a) CHT NPs and (b) EDTA-CHT NPs.

diameter of $100 \mathrm{~nm}$. Size and texture is affected when EDTA is added to the synthesis process: larger and smoother particles are obtained, possibly due to the aggregation effects previously discussed (Figure 5, column (b)). Encapsulation of imipenem on the latter system leads to NPs with an intermediate size and a rougher surface from that of EDTA-CHT NPs (Figure 5, column (c)). These images suggest that imipenem encapsulation leads to smaller nanoparticles when synthesis involves EDTA due to the higher molecular weight of the antibiotic in relation to water in case $\mathrm{B}$. 


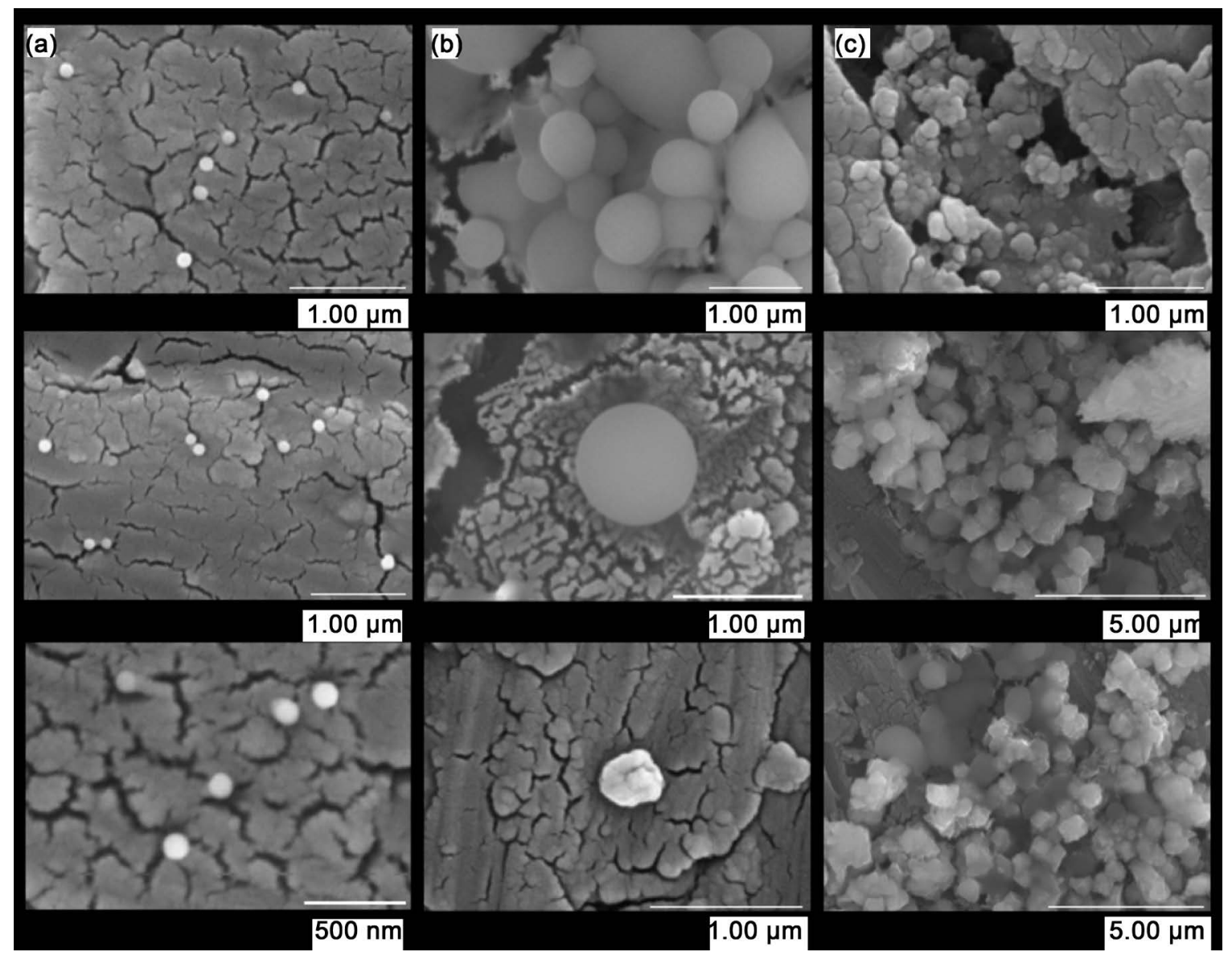

Figure 5. SEM images of different nanoparticle systems. Columns are as follows: (a) CHT NPs; (b) EDTA-CHT NPs; and (c) Imipenem-loaded EDTA-CHT NPs.

\subsection{In Vitro Drug Release Profile}

The in vitro cumulative release profile of imipenem from the CHT NPs is shown in Figure 6. Our observations indicate that $35.93 \%$ of the loaded imipenem was released within 24 hours of incubation in PBS. The release profile of imipenem loaded nanoparticles exhibits an initial burst release of about $16.64 \%$ in the first 0.25 hours followed by a slow release of $4.88 \%$ and $7.46 \%$ for between 8 and 20 hours (Figure 6). The rapid releasing process was mainly due to the nanoparticles surface drugs could easily diffuse in the initial time. The second phase was a relatively constant release ranging from hours 2 to 24 , which could be caused by the drugs diffused from the matrix.

\subsection{Dilution Assays}

In order to determine the range of EDTA concentrations at which growth of $P$. aeruginosa occur, a dilution assay with dilution ratio 0.5 and two replicate wells per sample was devised. EDTA concentrations with a maximum of $125 \mathrm{mM}$ were utilized in this assay, which is pivotal to avoid reporting significant growth inhibition by EDTA alone. Figure 7 shows that the minimum concentration of EDTA that does not inhibit bacterial growth is $1.957 \mathrm{mM}$. 


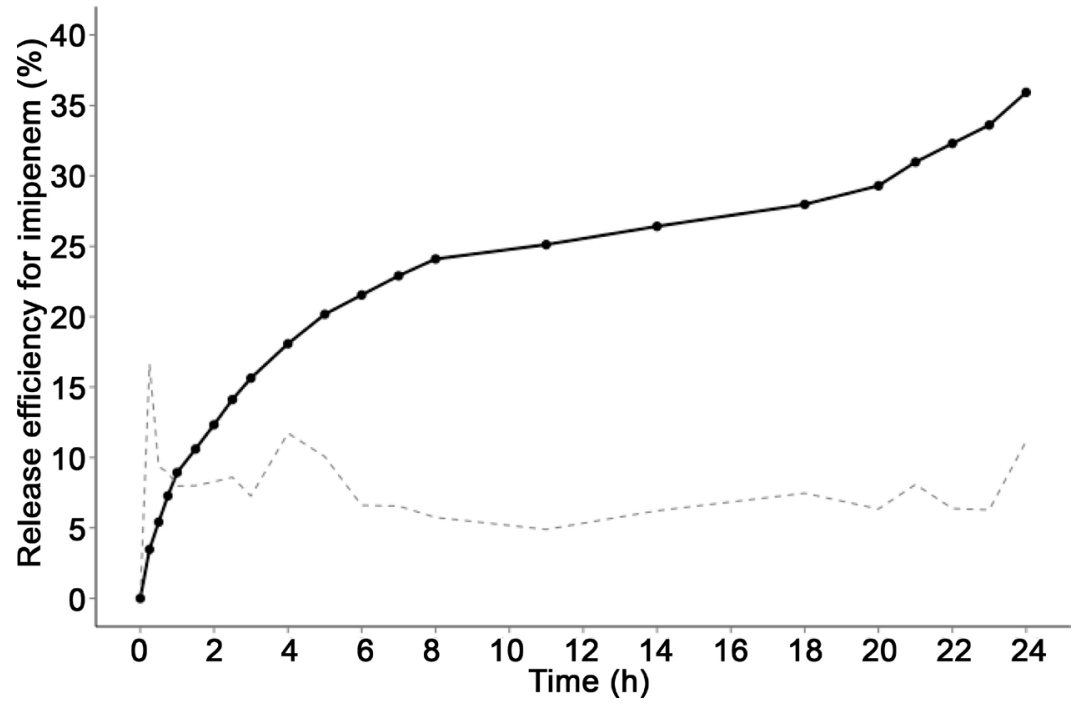

Figure 6. Release profile of imipenem from the CHT NP delivery system for $24 \mathrm{~h}$. The solid black line corresponds to the cumulative imipenem release efficiency profile and the dashed gray line to hourly measurements of release percentage.

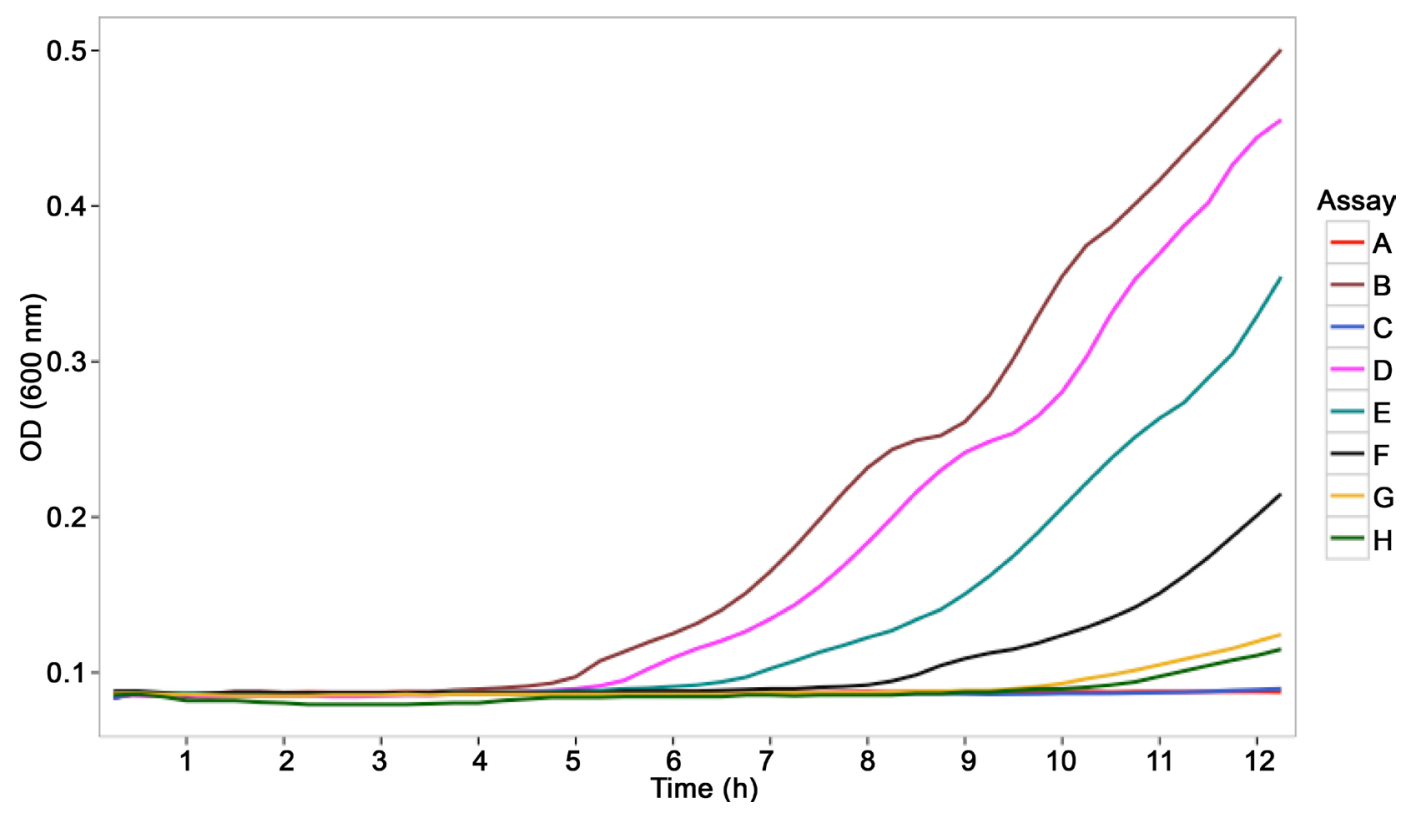

Figure 7. Effect of EDTA concentrations on P. aeruginosa AG1. Solid lines are as follows: (A) Lysogenic Broth medium, (B) P. aeruginosa AG1, (C) EDTA $125 \mathrm{mM}$, (D) EDTA $0.391 \mathrm{mM}$, (E) EDTA $0.781 \mathrm{mM}$, (F) EDTA $0.977 \mathrm{mM},(\mathrm{G})$ EDTA $1.563 \mathrm{mM}$, and (H) EDTA $1.957 \mathrm{mM}$.

An important consideration needs to be made in relation to the effective EDTA concentration in CHT NPs. A fraction of the EDTA molecules in the solution during functionalization process will attach to the surface of the nanoparticle, but not all of them. The concentration of EDTA carried by CHT NPs is a function of initial EDTA concentration, particle size and distribution of free amino groups on the surface. In general, if $r_{1}$ and $r_{2}$ represent the radii of two types of NPs where (without loss of generality) $r_{2}>r_{1}$, the relation between the surface areas is $\left(r_{2} / r_{1}\right)^{2}$. As SEM imaging reveals (Figure 5), CHT NPs that are 
functionalized with EDTA are larger than those loaded with imipenem. Considering that smaller nanoparticles are related to larger total available surface areas, it is necessary to compensate EDTA concentrations for the latter type of NPs. In that sense, we chose an initial EDTA concentration of $3 \mathrm{mM}$ for imipenem-loaded CHT NPs.

In order to determine the concentration of EDTA where synergistic effects were observed instead of EDTA leading to bacterial inhibition by itself, an assay with increasing EDTA concentrations was performed (Figure 8). As a result, a concentration of $3.0 \mathrm{mM}$ of EDTA was found to behave almost equally as that of nanoparticles without EDTA. Thus, this concentration was chosen for subsequent experiments. In the following assay, different concentrations of imipenem were used to determine the minimum concentration leading to bacterial inhibition of $P$. aeruginosa AG1, which is known to be resistant to carbapenems. $P$. aeruginosa PAO1 was used as control given its susceptibility to carbapenems. Results for the PAO1 strain (Figure 9(a)) indicate that even low concentrations of imipenem inhibited bacterial growth even at high dilutions. On the contrary, the AG1 strain (Figure 9(b)) was found to be most susceptible to a concentration of $20 \mathrm{mg} / \mathrm{ml}$ up to the sixth dilution. That concentration was hence selected to synthesize the final system.

The comparison between CHP NPs, EDTA-CHT NPs and imipenem-loaded EDTA-CHT NPs with a concentration of $20 \mathrm{mg} / \mathrm{ml}$ (complete system) was performed (Figure 10). The synergistic effect is observed in the curve for the complete system by sustaining its effectiveness up to the fifth dilution. The convexity of the curve is that of a multiplicative effect of the factors. The difference between CHT NPs and EDTA-CHT NPs is smaller, and the concavity indicates merely an additive effect. The latter suggests that the full system inhibits bacterial growth more effectively through a collection of synergistic mechanisms rather than each of them separately. In order to obtain the full picture for the fifth dilution in the

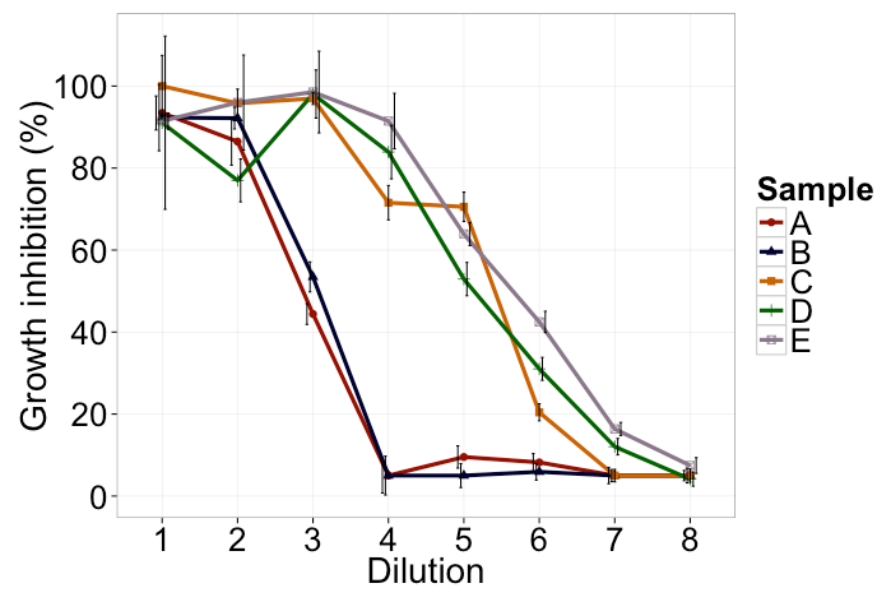

Figure 8. Effect of CHT-NPs functionalized with different EDTA concentrations (no antibiotic charge) on P. aeruginosa AG1. Solid lines are as follows: (A) CHT-NPs with no EDTA surface functionalization, CHT-NPs functionalized with EDTA concentrations of (B) $3.00 \mathrm{mM}$, (C) $21.50 \mathrm{mM}$, (D) $39.41 \mathrm{mM}$, and (E) $57.96 \mathrm{mM}$. 


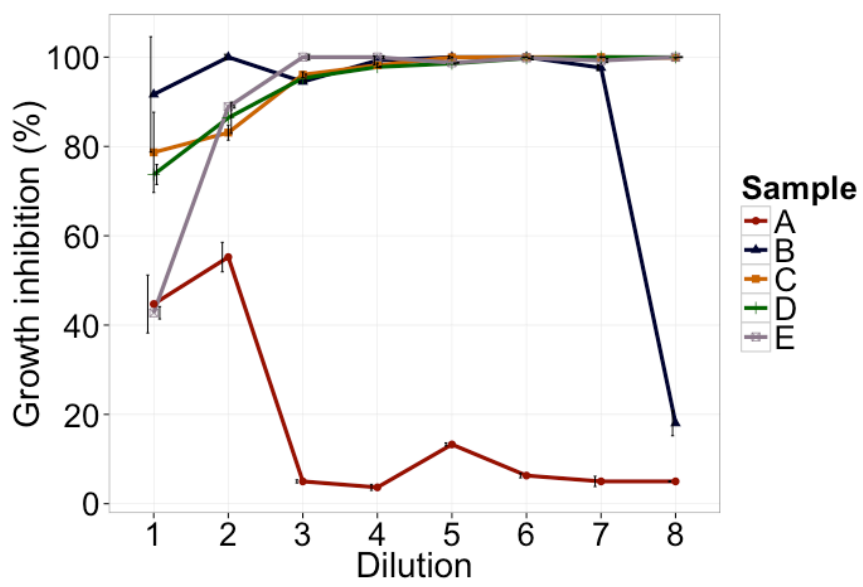

(a)

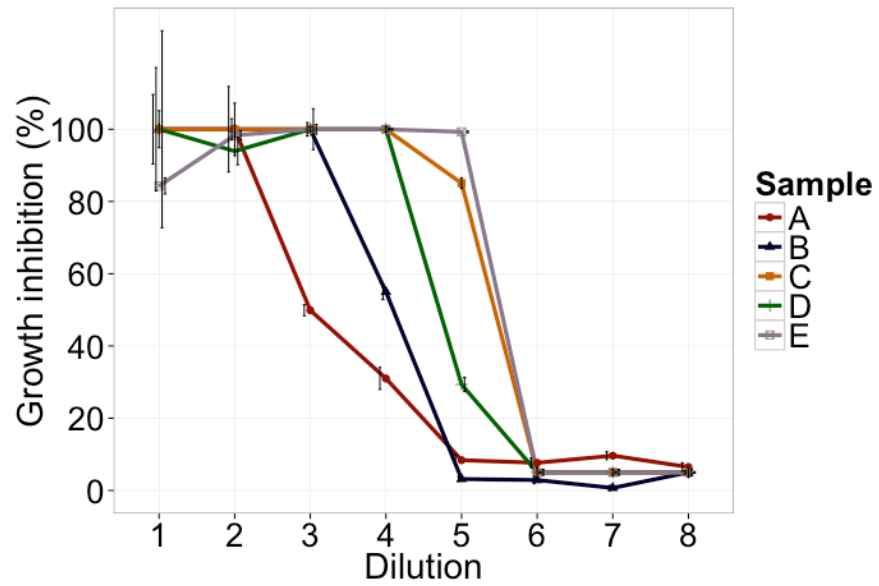

(b)

Figure 9. Dilution assays for imipenem-loaded EDTA-CHT NPs. PAO1 (a) and AG1 (b) strains were exposed to decreasing gradients of concentration. Lines are as follows: (A) NPs without imipenem; imipenem concentrations of (B) $1 \mathrm{mg} / \mathrm{ml}$, (C) $5 \mathrm{mg} / \mathrm{ml}$, (D) 10 $\mathrm{mg} / \mathrm{ml}$, and (E) $20 \mathrm{mg} / \mathrm{ml}$.

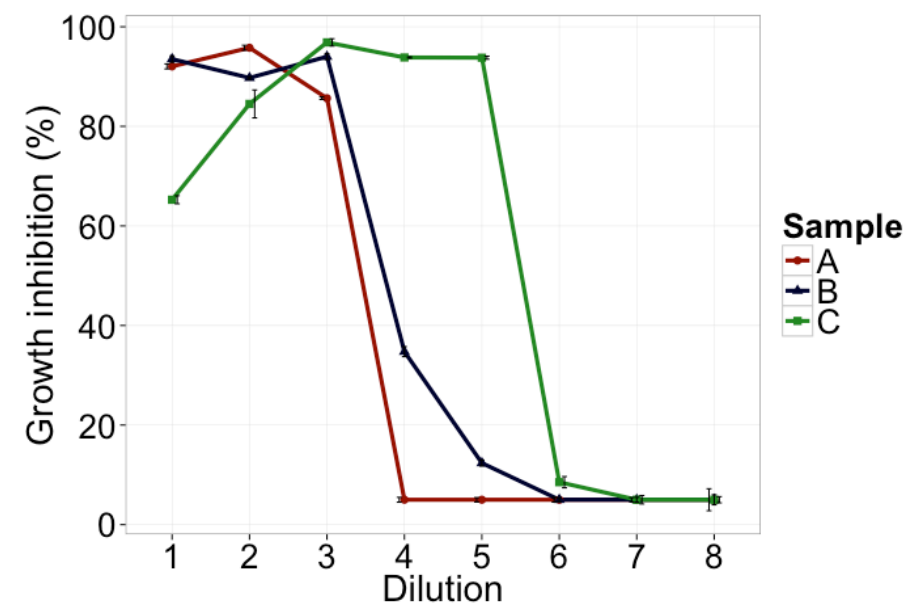

Figure 10. Dilution assays for imipenem-loaded EDTA-CHT NPs. P. aeruginosa AG1 strain was exposed to decreasing gradients of concentration. Lines are as follows: (A) CHT NPs, (B) EDTA-CHT NPs, (C) imipenem-loaded EDTA-CHT NPs. 


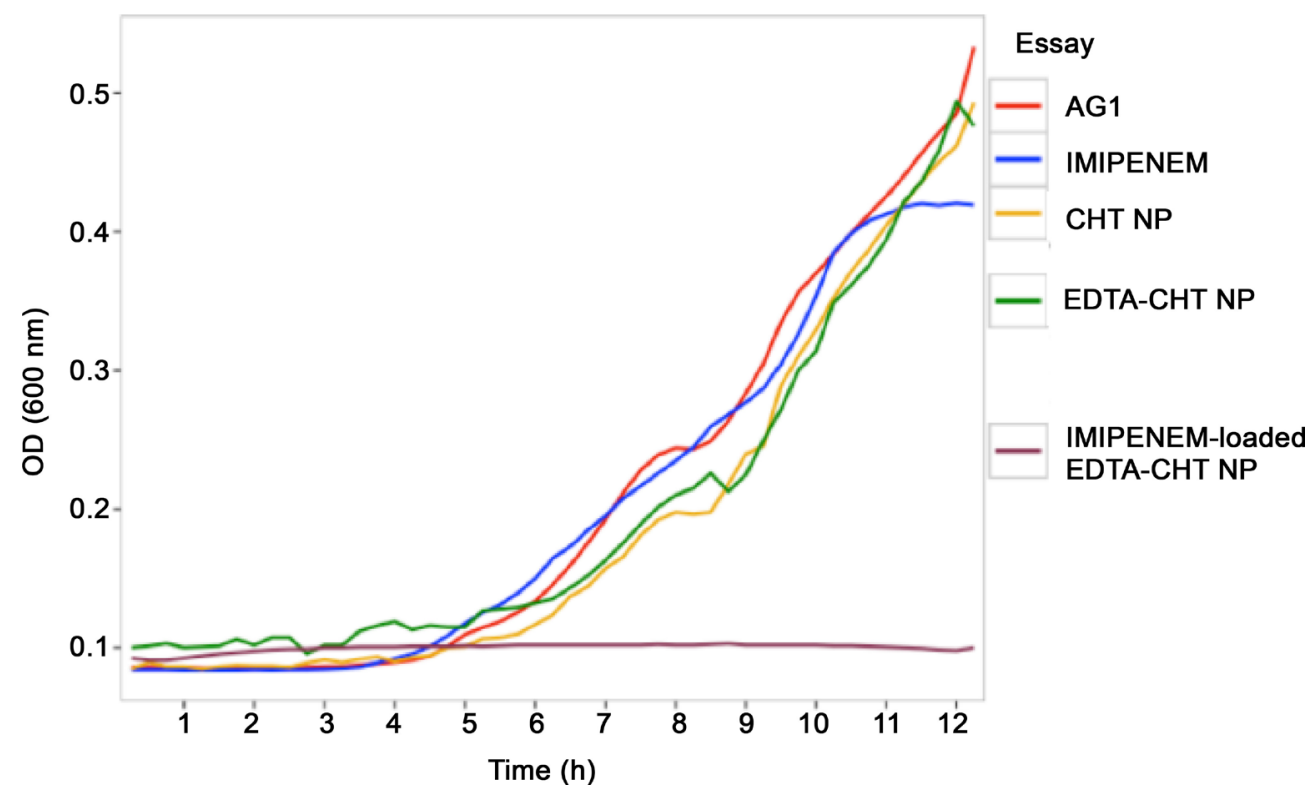

Figure 11. Growth curves corresponding to $P$. aeruginosa AG1 at dilution number five from Figure 10.

complete system, growth curves were obtained respectively for each nanoparticle system (Figure 11). The AG1 strain and AG1 under imipenem were used as controls. Both CHT NPs and EDTA-CHT NPs fail to inhibit bacterial growth at this point. The complete system (imipenem-loaded EDTA-CHT NPs) inhibited bacterial growth for the duration of the experiment.

\section{Conclusion}

The imipenem loaded CHT-EDTA NPs were successfully prepared based on ionic gelation by cross-linking with TPP to investigate the physicochemical properties of nanoparticles. The nanoparticles were stable and spherical in shape with a narrow size distribution. The release amounts of imipenem from the EDTA-CHT NPs suggested that nanoparticles have promising potential effect on antibiotic therapy.

\section{Acknowledgements}

The authors wish to acknowledge the Inter-University Fund for Higher Education (FEES) at the National Council of Rectors (CONARE) for financial support to this project through grant agreement No ACUERDO-VI-171-2014, the Research Center on Microscopic Structures (CIEMIC) at University of Costa Rica as well as, Maribel Chavarría (CIET), Rodrigo Muñoz (CENIBiot), Daniel Esquivel (CENIBiot) and Reinaldo Pereira (LANOTEC-CeNAT) for their collaboration and support with various aspects of this paper.

\section{References}

[1] Breidenstein, E., de la Fuente-Núñez, C. and Hancock, R. (2011) Pseudomonas aeruginosa: All Roads Lead to Resistance. Trends in Microbiology, 19, 419-426. https://doi.org/10.1016/j.tim.2011.04.005 
[2] Bonomo, R. and Szabo, D. (2006) Mechanisms of Multidrug Resistance in Acinetobacter Species and Pseudomonas aeruginosa. Clinical Infectious Diseases, 43, S49-S56. https://doi.org/10.1086/504477

[3] Khalifa, A., Moissenet, D., Thien, H. and Khedher, M. (2011) Virulence Factors in Pseudomonas aeruginosa: Mechanisms and Modes of Regulation. Annales De Biologie Clinique, 69, 393-403.

[4] Lister, P., Wolter, D. and Hanson, N. (2009) Antibacterial-Resistant Pseudomonas aeruginosa: Clinical Impact and Complex Regulation of Chromosomally Encoded Resistance Mechanisms. Clinical Microbiology Reviews, 22, 582-610. https://doi.org/10.1128/CMR.00040-09

[5] Master, R.N., Clark, R.B., Karlowsky, J.A., Ramirez, J. and Bordon, J.M. (2011) Analysis of Resistance, Cross-Resistance and Antimicrobial Combinations for Pseudomonas aeruginosa Isolates from 1997 to 2009. International Journal of Antimicrobial Agents, 38, 291-295. https://doi.org/10.1016/j.ijantimicag.2011.04.022

[6] Talbot, G.H., Bradley, J., Edwards, J.E., Gilbert, D., Sheld, M. and Barlett, J.G. (2006) Bad Bugs Need Drugs: An Update on the Development Pipeline from the Antimicrobial Availability Task Force of the Infectious Diseases Society of America. Clinical Infectious Diseases, 42, 657-668. https://doi.org/10.1086/499819

[7] Coates, A.R. (2012) Antibiotic Resistance. Handbook of Experimental Pharmacology. University of London. Springer, Berlin.

https://doi.org/10.1007/978-3-642-28951-4

[8] Walsh, C. (2003) Antibiotics. Action, Origins, Resistance. ASM Press, Washington DC.

[9] Toval, F., Guzmán-Marte, A., Madriz, V., Somogyi, T., Rodriguez, C. and García, F. (2014) Predominance of Carbapenem-Resistant Pseudomonas aeruginosa Isolates Carrying blaimp and blaviм Metallo-Lactamases in a Major Hospital in Costa Rica. Journal of Medical Microbiology, 64, 37-43. https://doi.org/10.1099/jmm.0.081802-0

[10] Boonsongrit, Y., Mitrevej, A. and Mueller, B. (2006) Chitosan Drug Binding by Ionic Interaction. European Journal of Pharmaceutics and Biopharmaceutics, 62, 267-274. https://doi.org/10.1016/j.ejpb.2005.09.002

[11] El-Sharif, A. and Hussain, M. (2010) Chitosan-EDTA New Combination Is a Promising Candidate for Treatment of Bacterial and Fungal Infections. Current Microbiology, 62, 739-745. https://doi.org/10.1007/s00284-010-9777-0

[12] Cetin, M., Aktas, Y., Vural, I., Capan, Y., Dogan, L., Duman, M. and Dalkara, T. (2007) Preparation and In Vitro Evaluation of bFGF-Loaded Chitosan Nanoparticles. Drug Delivery, 14, 525-529. https://doi.org/10.1080/10717540701606483

[13] Fàbregas, A., Miñarro, M., García-Montoya, E., Pérez-Lozano, P., Carrillo, C., Sarrate, R., Sánchez, N., Ticó, J. and Suñé-Negre, J. (2013) Impact of Physical Parameters on Particle Size and Reaction Yield When Using the Ionic Gelation Method to Obtain Cationic Polymeric Chitosan-Tripolyphosphate Nanoparticles. International Journal of Pharmaceutics, 446, 199-204. https://doi.org/10.1016/j.ijpharm.2013.02.015

[14] Janes, K., Fresneau, M., Marazuela, A., Fabra, A. and Alonso, M. (2001) Chitosan nanoparticles as Delivery Systems for Doxorubicin. Journal of Controlled Release, 73, 255-267. https://doi.org/10.1016/S0168-3659(01)00294-2

[15] Bernkop-Schnürch, A. and Scerbe-Saiko, A. (1998) Synthesis and In Vitro Evaluation of Chitosan-EDTA-Protease-Inhibitor Conjugates Which Might Be Useful in Oral Delivery of Peptides and Proteins. Pharmaceutical Research, 15, 263-269. 
https://doi.org/10.1023/A:1011970703087

[16] Singh, K., Suri, R., Tiwary, A. and Rana, V. (2013) Exploiting the Synergistic Effect of Chitosan-EDTA Conjugate with MSA for the Early Recovery from Colitis. International Journal of Biological Macromolecules, 54, 186-196.

https://doi.org/10.1016/j.ijbiomac.2012.12.026

[17] Guzmán-Marte, A.E. (2013) Localización, transferabilidad y tipificación de los genes bla 1 м y blavim en Pseudomonas aeruginosa AG1 resistente a carbapenems. Tesis de Maestría, Sistema de Estudios de Posgrado, Universidad de Costa Rica.

[18] Ajun, W., Yan, S., Li, G. and Huili, L. (2009) Preparation of Aspirin and Probucol in Combination Loaded Chitosan Nanoparticles and in Vitro Release Study. Carbohydrate Polymers, 75, 566-574. https://doi.org/10.1016/j.carbpol.2008.08.019

[19] Ji, J., Hao, S., Wu, D., Huang, R. and Xu, Y. (2011) Preparation, Characterization and in Vitro Release of Chitosan Nanoparticles Loaded with Gentamicin and Salicylic Acid. Carbohydrate Polymers, 85, 803-808. https://doi.org/10.1016/j.carbpol.2011.03.051

[20] Hanaor, D., Michelazzi, M., Leonelli, C. and Sorrell, C. (2012) The Effects of Carboxylic Acids on the Aqueous Dispersion and Electrophoretic Deposition of $\mathrm{ZrO}_{2}$. Journal of the European Ceramic Society, 32, 235-244. https://doi.org/10.1016/j.jeurceramsoc.2011.08.015

[21] Gan, Q. and Wang, T. (2007) Chitosan Nanoparticle as Protein Delivery Carrier-Systematic Examination of Fabrication Conditions for Efficient Loading and Release. Colloids and Surfaces B: Biointerfaces, 59, 24-34. https://doi.org/10.1016/j.colsurfb.2007.04.009

[22] Rana, R., Murthy, V., Yu, J. and Wong, M. (2005) Nanoparticle Self-Assembly of Hierarchically Ordered Microcapsule Structures. Advanced Materials, 17, 1145-1150.

[23] Mohammadpour, D.N., Eskandari, R., Avadi, M.R., Zolfagharian, H., Mir Mohammad Sadeghi, A. and Rezayat, M. (2012) Preparation and in Vitro Characterization of Chitosan Nanoparticles Containing Mesobuthus eupeus Scorpion Venom as an Antigen Delivery System. Journal of Venomous Animals and Toxins Including Tropical Diseases, 18, 44-52. 